critical pH on adding the acidic dithionite, LAURELL rcleased carbon dioxide from the serum by deep freezing for 24 hours, followed by thawing. In the present investigation the procedure followed was to buffer the reagent to $\mathrm{pH}$ approximately 7.5 by adding Tris, so that even fresh serum could be used without any risk.
The makrolon tubes supplicd arc packed in dust-tight containers and can be used immediatcly so that timeconsuming cleaning of glass ware is avoided.

The use of phenol results in a complete deprotcinisation during the heating and the supernatant is optically clear.

\title{
References
}

1. Hunkr, R. J., C. H. Sougl and N. Chinmori, Clin. chim. Acta (Amsterdnm) 3, 523 (1958). - 2. Ramsay, W. N. M., Clin. chim. Actn (Ansterdam) 2, 221 (1957). - 3. Laurzil., C.-B., Actn physiol. Scand., 14, suppl. 46 (1947). - 4. PI.ODIN, P., Dextran gels and their applications in gel filtration. 'Thesis, Uppsala (1962). 5. Barber, A., C. Demipster and N. Anderson, Clin. chim. Acta
(Amsterdam) 8, 143 (1963). - 6. London, M. Clin. Chem. New York 10, 789 (1964). - 7. PArruck, R. J., and R. E. THulers, Clin. Chem. New York 9, 283 (1963). - 8. Giovannibllo, 'Tir. J., and TH. Peters, Standard methods of clinical chemistry, $\mathrm{IV}^{r}$ p. 139. Hrsg. D. Scligson, Academic Press, New York (1963).

\section{Qualitative Veränderungen verschiedener jodierter phenolischer Aminosäuren im Laufe einer alkoholischen Extraktion aus wäßrigen Lösungen}

\author{
Von E. Zappr und G. Hoppls \\ Aus der 1. Mediziniscben Klinik des Städtiscben Auguste-Viktoria-Krankenhauses Berlin-Scljöneberg \\ (Direktor: Prof. Dr.K. H. Pfeffer)
}

(13ingegangen am 10. Oktober 1967)

Es werden die chemischen Veränderungen, die einige jodicrte phenolische Amninosäuren in Verlaufe ciner Extraktion aus wäßrigen Lösungen erfahren, untersucht. Dic durchgeführten chemischen und dünnschichtchromatographischen Kontrollen \%eigen, da $\beta$ dic prozentualen Verluste der $\Lambda$ usgangssubstanzen meistens relativ geringfügig sind. Die im Verlauf der Extraktion neugebildeten Verbindungen lassen sich, mit nur einer Ausnahme, als jodierte phenolische Aminosäuren identifizieren.

The qualitative changes which occur in a number of iodinated phenolic amino acids during alcoholic extraction from aqueous solutions, were investigated.

Thin-layer chromatographic controls showed that in most cases the decomposition of the starting matcrials is relatively sinall. The new compounds formed during the extraction can be classified, with one exception, as iodinated phenolic amino acids.

Aufgrund früherer Untersuchungen über dic Verteilungskocffizienten verschicdencr jodierter phenolischer Aminosäuren in ciner Reihe wäßrig-organischer Zweiphascn-Systeme (1) wurde eine Methode entwickelt, diese Substanzen aus dem Serum zu gewinnen. Die hohe Ausbeute ermöglicht nach weitergehender Rcinigung des Extraktes in Verbindung mit cinem jüngst beschricbenen dünnschichtchromatographischen System (2) die Trennung und Identifikation zirkulierender Schilddrüsenhormone und Derivate $(3,4)$.

Die Frage der eventuellen Zersetzung der jodierten Substanzen im Verlauf der Extraktion wird hier mittels rciner wäßriger Lösungen verschiedener Jodtyrosinc und Jodthyronine, die der Extraktion unterworfen wurden, näher untersucht. Dic Kontrollen wurden mit Hilfe der Dünnschichtchromatographic durchgeführt. Dic Anwendung einer relativ cinfachen Technik - auch hier beschrieben - gestattet die Auswertung der Intensität der vorhandenen Flecken auf den Chromatogrammen durch Extinktionsmessung.

\section{Methodik}

Als Versuchssubstanzen wurden folgende jodierte plienolische Aminosäuren verwendet: 3-Monojod- und 3,5-Dijod-L-Tyrosin, 3-Monojod-, 3,5-Dijod-, 3,3',5-Trijod- und 3,3',5,5'-Tetrajod-DLthyronin (MIT, DIT, $T_{1}, T_{2}, T_{3}, T_{4}$ ). Dicsc Substanzen wurden init $0,05 \mathrm{~N} \mathrm{NaOH}(0,5 \mathrm{mg}$ pro $\mathrm{m} /)$ gelöst und getrennt bearbeitet.

\section{Extraktionsverfaljren}

Dic Extraktion wurde folgendermaßen durchgeführt: $6 \mathrm{~m} / \mathrm{von}$ jeder der angesetzten Lösungen (glcich $3 \mathrm{mg}$ Versuchssubstanz) wurden in Zentrifugenröhrchen mit Schliff (ettva $65 \mathrm{~m} /$ Fassungsvermögen) mit $0,5 \mathrm{~m} / 1 \mathrm{M}$ Propylthiouracillösung verset/t, mit cinigen Tropfen konz. Essigsäure auf $\mathrm{pH}<5$ eingestellt und $5 \mathrm{Min}$. mit $15 \mathrm{~m} /$ Methanol an Rückflußkühler gekocht.

Dic wäßrig-alkoholische Phase wird nun mit $2 \mathrm{~g}$ eincr Mischung Natriumsulfat-Aktivkohle 200:1 geschüttelt, zentrifugicrt und nach Abtrennung des Rückstandes in cin anderes Schliffröhrchen überführt und am Rotationsverdainpfer bei Unterdruck und 55-60 eingeengt. Der Unterdruck wurde durch cine Wasserstrahlpumpe erecugt. Der trockene Rückstand wird mit $3 \mathrm{~m} / 0,05 \mathrm{~N} \mathrm{NaOH}$ aufgenommen und in ein Zentrifugenröhrchen mit einem Fassungsvermögen von ctwa $45 \mathrm{n} /$ gebrncht. Das vorherige Röhrchen wird mit $3 \mathrm{~m} / 0,05 \mathrm{~N}$ NaOH nachgespült und dieser Anteil mit dem andcren gemischt. Die wäßrige Lösung wird nach Kontrolle mit 
Indikatorpapier $(\mathrm{pH}>10)$ einer Extraktion mit $7 \mathrm{~m} /$ Petroläther (Siedebereich $30-50^{\circ}$ ) durch Schütteln und Zentrifugieren untervorfen. Nach der Entfernung der überstehenden Phase.wird in dersclben Weise eine Extraktion mit Chloroform durchgeführt. Dieses wird ebenfalls nach Zentrifugieren größtenteils abgesaugt und der Rest bei Unterdruck abgedampft.

Der $\mathrm{pH}$ der wäßrigen Phase wird mit konz. Essigsäure auf einen Wert $<5$ eingestellt. Die wäßr. Phase wird mit $10 \mathrm{~m} /$ redestilliertem n-Butanol geschüttelt und zentrifugiert. Die überstehende Phase wird sorgfältig abgesaugt und aufgehoben; die wäßrige wird mit $4 \mathrm{~m} /$ Butanol wieder kurz geschüttelt und zentrifugiert. Man saugt ebenso die alkoholische Phase ab, die mit der ersten zusammen in ein Zentrifugenglas $(25 \mathrm{~m} /)$ überführt wird.

Der butanolische Extrakt wird mit $5 \mathrm{~m} /$ Wasser geschüttelt. Nach Zentrifugieren und Absaugen des Wassers wird $1 \mathrm{~g}$ Natriumsulfat-Aktivkohlegemisch zugegeben, wieder geschüttelt und zentrifugiert. Der Extrakt wird in einen $10 \mathrm{ml}$ Meßkolben gebracht und mit Butanol auf dieses Volumen ergänzt. Bei 100 proz. Extraktion enthält $1 \mathrm{~m} l 300 \mu \mathrm{g}$ Substanz.

\section{Dünnschicbtcbromatographie}

Für die Trennung und Identifikation der infrage kommenden Substanzen wurde vorwiegend ein aufsteigendes dünnschichtchromatographisches System gewählt, das aus Cellulosepulver mit Gipszusatz als Adsorptionsmittel und Aceton/0,5N Essigsäure 2:8 $(\mathrm{V} / \mathrm{V})$ als mobile Phase bestand. 5 Glasplatten $(20 \times 20 \mathrm{~cm})$ wurden mit einer Mischung von 25 g MN 300 G (Macherey, Nagel \& Co. Düren) und $150 \mathrm{~m} l$ Wasser beschichtet und nach Trocknung im Exsikkator über Blau-Gel aufbewahrt. Die $R_{\mathrm{F}}$-Werte der untersuchten Substanzen in diesem System sind folgende: MIT, 0,82; DIT, 0,$71 ; T_{1}, 0,75 ; T_{2}, 0,65 ; T_{3}, 0,36 ; T_{4}, 0,18$. Die maximale Kapazität des Systems (Schichtstärke etwa $1 \mathrm{~mm}$ ) beträgt 5-10 $\mu \mathrm{g}$ jeder dieser Substanzen: Beim Auftragen größerer Mengen läßt die Trennfähigkeit in zunehmendem Maße nach. Dementsprechend wurde bei den butanolischen Extrakten ein Auftragsvolumen von $30 \mu l$ (maximal $9 \mu \mathrm{g}$ Substanz) nicht überschritten. Die Empfindlichkeit des Jodnachweis-Reagenz gestattet die Erfassung von $0,01-0,05 \mu \mathrm{g}$ dieser Jodverbindungen.

Zwecks weiterer Identifizierung der nach der Extraktion vorkommenden Verbindungen wurden gegebenenfalls parallele Ansätze der Extrakte mit Kontrollsubstanzen in anderen dünnschichtchromatographischen Systemen untersucht.

\section{Anfärbung}

Für die allgemeine Sichtbarmachung der jodierten Verbindungen leistete das Ferrichlorid-(Kalium)Ferricyanid-Arsenige-Säure(FFCA-)Reagenz ${ }^{1}$ ) nach GMELIN und VIRTANEN (5) ausgezeichnete Dienste. Nach eigener Modifikation für die Dünnschichtchromatographie (6) ist der Nachweis bis $0,05-0,01 \mu$ g jeder der geprüften Jodverbindungen möglich. Zur Bestätigung des Jodgehaltes der durch das Gmelin- und Virtanen-Reagenz dargestellten Flecke wurde die von PostMEs vorgeschlagene Modifikation, Ferrichlorid(Kalium)Ferricyanid-(FFC-)Reagenz angewendet. Die jodierten Substanzen sind FFCA-positiv aber FFC-negativ, während die Substanzen, die fälschlicherweise positiv reagieren, auch FFCpositiv sind (7).

Zum Nachweis von Aminosäuren und phenolischen Gruppen wurden Ninhydrin bzw. Pauly's Reagenz verwendet (8), dessen Empfindlichkeit jedenfalls um zwei Dezimalstellen höher als bei der Gmelin- und Virtanen-Reaktion liegt.

\section{Auswertung der Chromatogramme}

Von den unmittelbar nach der Entwicklung fotografierten Chromatogrammen wurden außer schwarz-weißen Abzügen zur Dokumentation der Ergebnisse andere auf transparentem Film angefertigt. Diese wurden, in Streifen zerschnitten, in einem Elphor Integraph (Dr. Bender \& Dr. Hobein, München-Karlsruhe-

1) Abkürzungen: FFCA = Ferrichlorid-(Kalium)Ferricyanid-Arsenige-Säure-Reagenz für jodierte Thyronine und Tyrosine nach GMELIN und VirTANEN; FFC = Modifiziertes Gmelin und VIRTANEN-Reagenz nach PostMES, Ferrichlorid-(Kalium)Ferricyanid.
Zürich) ausgewertet und zu einer Kurve integriert (Abb. 1). Die von jedem Streifen erhaltenen absoluten Extinktionswerte wurden in prozentuale Beziehungen zueinander gesetzt. Diese Technik ist viel leichter durchführbar als die bereits beschriebene direkte Auswertung der Schicht nach Ablösung von der Glasplatte (9).

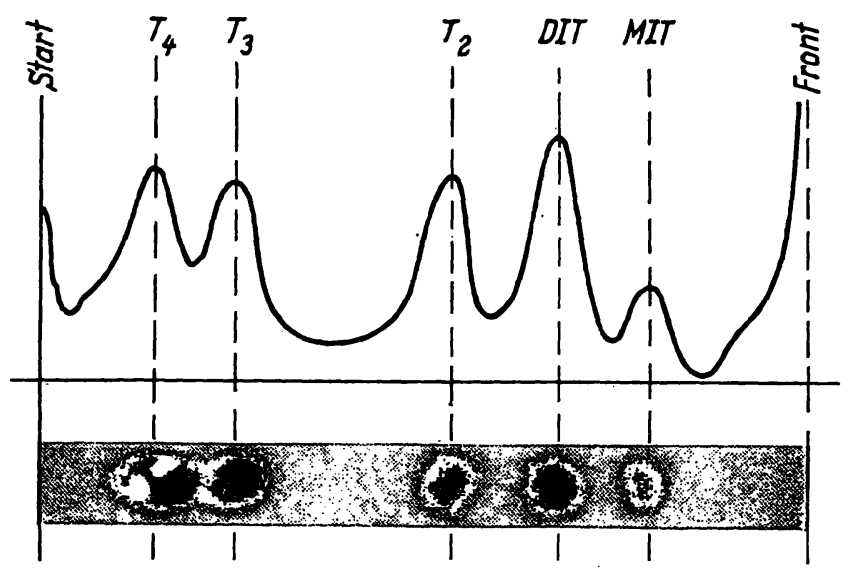

Abb. 1

Densitometrische Auswertung eines Chromatograms
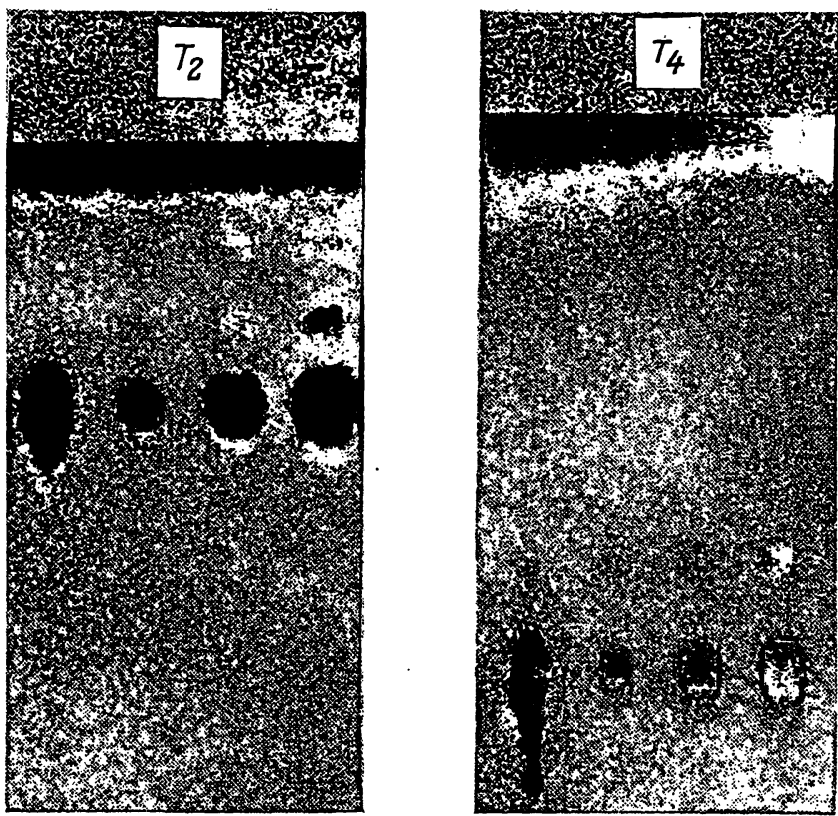

Abb. 2

Chromatogramme butanolischer Lösungen von $T_{2}$ und $T_{4}$ im Vergleich zu den Chromatogrammen der Ausgangslösungen

In beiden Fällen links das Chromatogramm der Ausgangslösung ( $5 \mu \mathrm{g}$ Substanz), rechts daneben je drei Chromatogramme der erhaltenen butanolischen Lösungen $(10,20$ und $30 \mu l)$.

\section{Ergebnisse}

Die Beobachtung der erhaltenen Platten zeigt eine ständige Konkordanz der Flecke der Kontrollchromatogramme mit denen der entsprechenden butanolischen Extrakte, die homogen, gut begrenzt und reproduzierbar sind. In einigen Fällen ist die Neubildung von Substanzen im Laufe der Extraktion durch das Auftauchen anderer Flecken außer denen der Hauptsubstanz auf dem Chromatogramm erkennbar (Abb. 2). Die $R_{\mathrm{F}}$-Werte jedes Fleckens der butanolischen Extrakte, sowie ihre prozentualen Anteile an den Chromatogrammen sind in Tabelle 1 dargestellt. Wie aus dem Vergleich der Werte 
Tab. 1

Densitometrische Auswertung, $R_{\mathrm{F}}$-Werte und Identifikationsreaktionen der Flecke der butanolischen Extrakte $(30 \mu l)$.

Ganz links, senkrecht, die Standardsubstanzen nach ihrem chromatographischen Verhalten eingeordnet. Rechts davon, auch senkrecht, die erhaltenen Ergebnisse der Chromatogramme der butanolischen Lösungen. Jedes ausgefüllte Kästchen stellt einen Fleck dar, dessen errechneter prozentualer Anteil, $R_{\mathrm{F}}$-Werte und Identifikationsreaktionen in dieser Reihenfolge eingetragen sind.

\begin{tabular}{|c|c|c|c|c|c|c|}
\hline \multirow[b]{2}{*}{. } & MIT & DIT & $T_{1}$ & $T_{2}$ & $T_{3}$ & $T_{4}$ \\
\hline & & & & $\begin{array}{l}\quad 12,0 \% \\
\quad R F=0,87 \\
\text { FFCA } \\
\text { FFC } \\
\text { Ninhyd. } \\
\text { Pauly's }\end{array}$ & & \\
\hline$\underset{R_{\mathrm{F}} 0,82}{\mathrm{MIT}}$ & $\begin{array}{l}100,0 \% \\
R_{\mathrm{F}} 0,82\end{array}$ & $\begin{array}{r}13,7 \% \\
R_{\mathrm{F}} 0,81\end{array}$ & & & & \\
\hline $\begin{array}{l}\text { FFCA } \\
\text { FFC } \\
\text { Ninhyd. } \\
\text { Pauly's }\end{array}$ & $\begin{array}{l}\text { FFCA } \\
\text { FFC } \\
\text { Ninhyd. } \\
\text { Pauly's }\end{array}$ & $\begin{array}{l}\text { FFCA } \\
\text { FFC } \\
\text { Ninhyd. } \\
\text { Pauly's }\end{array}+$ & & & & \\
\hline $\begin{array}{c}\mathrm{T}_{1} \\
R_{\mathrm{F}} 0,75\end{array}$ & & & $\begin{array}{c}87,6 \% \\
R_{\mathrm{F}} 0,75\end{array}$ & $\begin{array}{c}24,4 \% \\
R_{\mathrm{F}} 0,76\end{array}$ & & \\
\hline $\begin{array}{l}\text { FFCA } \\
\underset{\text { Ninhyd. }}{\text { NFauly's }}\end{array}+$ & & & $\begin{array}{l}\text { FFCA } \\
\text { FFC } \\
\text { Paulyds }\end{array}+$ & $\begin{array}{l}\text { FFCA } \\
\text { FFC } \\
\text { Paulyds }\end{array}+$ & & \\
\hline $\begin{array}{c}\text { DIT } \\
R_{\mathrm{F}} 0,71\end{array}$ & & $\begin{array}{c}86,3 \% \\
R_{F} 0,71\end{array}$ & & & & \\
\hline $\begin{array}{l}\text { FFCA } \\
\text { FFC } \\
\text { Ninhyd. } \\
\text { Pauly's }\end{array}+$ & & $\begin{array}{l}\text { FFCA } \\
\text { FFC } \\
\text { Ninhyd. } \\
\text { Pauly's }\end{array}$ & & & & \\
\hline$R_{\mathrm{F}} \mathrm{T}_{2} 0,65$ & & & $\begin{array}{c}12,4 \% \\
R_{\mathrm{F}} 0,64\end{array}$ & $\begin{array}{c}63,6 \% \\
R_{\mathrm{F}} 0,65\end{array}$ & & \\
\hline $\begin{array}{l}\text { FFCA } \\
\text { FFC } \\
\text { Ninhyd. } \\
\text { Pauly's }\end{array}$ & & & $\begin{array}{l}\text { FFCA } \\
\text { FFC } \\
\text { Ninhyd. } \\
\text { Pauly's }\end{array}+$ & $\begin{array}{l}\text { FFCA } \\
\text { Ninhyd. } \\
\text { Pauly's }\end{array}+$ & & \\
\hline$R_{\mathrm{F}} \mathrm{T}_{\mathbf{3}} \mathbf{3 6}$ & & & & & $\begin{array}{l}1.00,0 \% \\
R_{F} 0,35\end{array}$ & $\begin{array}{r}22,4 \% \\
R_{\mathrm{F}} 0,35\end{array}$ \\
\hline $\begin{array}{l}\text { FFCA } \\
\text { FFC } \\
\text { Ninhyd. } \\
\text { Pauly's }\end{array}+$ & & & & & $\begin{array}{l}\text { FFCA } \\
\text { FFC } \\
\text { Ninhyd. } \\
\text { Pauly's }\end{array}+$ & $\begin{array}{l}\text { FFCA } \\
\text { FFC } \\
\text { Paulyd. }\end{array}$ \\
\hline$R_{\mathrm{F}}^{\mathrm{T}} \mathrm{O}, 18$ & & & & & & $\begin{array}{c}77,6 \% \\
R_{\mathrm{F}} 0,19\end{array}$ \\
\hline $\begin{array}{l}\text { FFCA } \\
\text { FFC } \\
\text { Ninhyd. } \\
\text { Pauly's }\end{array}+$ & & & & & & $\begin{array}{l}\text { FFCA } \\
\text { FFC } \\
\text { Ninhyd. } \\
\text { Pauly's }\end{array}$ \\
\hline
\end{tabular}

hervorgeht, entsprechen die $R_{\mathrm{F}}$-Werte der Hauptflecke der butanolischen Extrakte mit Genauigkeit denen der Ausgangssubstanzen. Die neugebildeten Verbindungen, die in wesentlich kleineren Mengen auf den meisten der Chromatogramme vorkommen, haben mit nur einer Ausnahme $R_{\mathrm{F}}$-Werte, die denen irgendwelcher KontrollSubstanzen entsprechen. Da eine weitere Untersuchung -ergab, daß alle dargestellten Substanzen - außer der oben erwähnten Ausnahme - Aminosạuren sind, die phenolis'che Gruppe besitzen (Tab. 1), liegt es nahe, anzunehmen, daß diese unbekannten Verbindungen mit den Kontrollsubstanzen identisch sind. Das chromatographische Verhalten der so klassifizierten Substanzen und der entsprechenden Kontrollen, die in parallelen Ansätzen in anderen dünnschichtchromatographischen Systemen nicht abwichenen, bestätigte diese Auffassung. Von chemischen Veränderungen im Laufe der Extrak= tion sind hauptsächlich $T_{2}$ und $T_{4}$ betroffen. Der Anteil der ersten wird durch die Darstellung von z̈wei neuen Flecken auf dem Chromatogramm der butanolischen Lösung auf 63,6\% erniedrigt (Abb. 3). Der Ausfall beträgt bei $\mathrm{T}_{4} 22,4 \%$ mit einer entsprechenden Neubildung von $\mathrm{T}_{3}$. Bei DIT liegt der Ausfall bei 13,7\%, bei $\mathrm{T}_{1}$ bei $12,4 \%$; MIT und $\mathrm{T}_{3}$ sind nach der Extraktion unverändert.

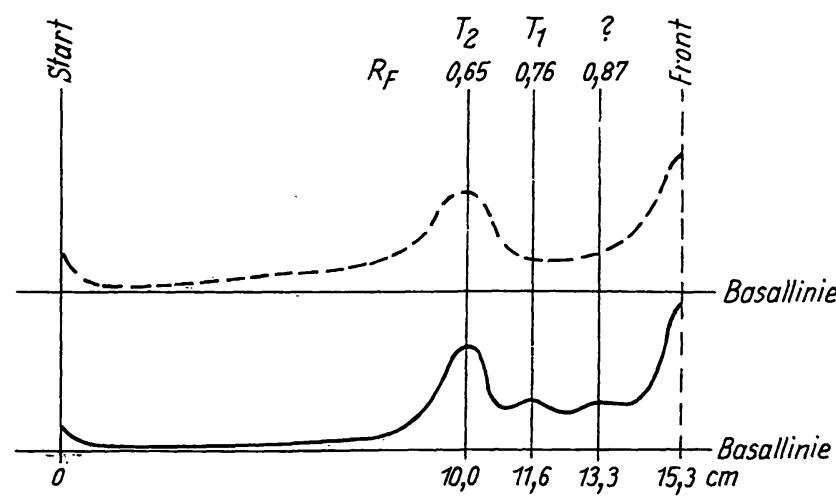

Abb. 3

Extinktionskurven der Chromatogxamme der $T_{2}$-Lösungen, vor und nach der Extraktion, vergleichsweise dargestellt.

$$
\begin{aligned}
& \text { - Ausgangslösung } \\
& \text { butanolischer Extrakt }
\end{aligned}
$$

\section{Diskussion}

Die Zersetzung der jodierten Verbindungen im Laufe des Verfahrens sowie die Bildung von Artefakten, von diesen Substanzen ausgehend, sind offene Fragen bei den meisten Extraktionsmethoden von Schilddrüsenhormonen aus Serum oder Gewebe $(10,11)$. Die Untersuchung reiner wäßr. Lösungen von Jodophenolen 
unter denselben Bedingungen und die Analyse der erhaltenen Extrakte gestatten einen objektiven Einblick in das Problem der chemischen Umwandlungen der Ausgangssubstanzen während des Arbeitsganges.

So wurde hier festgestellt, daß die meisten der geprüften jodierten Tyrosine und Thyronine die hier beschriebene Methode ohne große Änderungen überstehen. Die Identität der zurückgewonnenen Substanzen konnte durch übereinstimmende Ergebnisse der chemischen und chromatographischen Analysen mit nur einer Ausnahme zufriedenstellend aufgeklärt werden. Die prozentualen
Verluste der Ausgangssubstanzen ließen sich mit Hilfe der indirekten Auswertung der Chromatogramme gut erfassen.

Von quantitativen Aussagen wurde Abstand genommen: Diese werden als zweite systematische Untersuchung der Leistungen dieser Aufarbeitungsmethode mittels chemischer und isotopischer Methoden aufgeklärt.

Herrn MrChael Schmidr möchten wir für seine technische Hilfe danken. Frl. H. KerLer sind wir für die Hilfe am Manuskript zu Dank verpflichtet.

\section{Literatur}

1. Zappr, E., J. Chromatogr. 30, 611 (1967). - 2. Gries, G., K. H. Pfeffer und E. ZAppi, Klin. Wschr. 43, 515 (1965). - 3. Hoppe, G., E. ZAPpi und G. Gries, Nucl.-Med. (Stuttgart) 6, 44 (1967). - 4. ZAppi, E. und G. Hoppe, diese Z. 5, 209 (1967). - 5. GMelin, R. und A. I. Virtanen, Acta chem. Scand. 13, 1469 (1959). - 6. Zappi, E., J. Chromatogr. 31, 241 (1967). - 7. Postmes, Th., Clin. chim. Acta (Amsterdam) 10, 581 (1964). - 8. WALdr, D., in Dünn- schichtchromatographie, Hrsg. E. Stahl, S. 501. Springer-Verlag, Berlin, Göttingen, Heidelberg (1962). - 9. Morrn, R. J., Clin. chim. Acta (Amsterdam) 13, 395 (1966). - 10. Escobar DEL Rey, F., G. Morreate de Castro und A. A. Kassenaar, Scand. J. Clin. Laborat. Invest. 8, 243 (1956). - 11. Tóng, W. und I. L. Chaikoff, J. biol. Chemistry 232, 939 (1958).

Dr. E. Zappi

1 Berlin 41

Rubensstraße 125

\section{KURZMITTEILUNGEN}

\section{Über den Nachweis des Methamphetamins $\left(\right.$ Pervitin $\left.^{1}\right)$ ) im menschlichen Speichel II. Mitteilung zum Problem des Dopings')}

Von D. Clasing, H. Alfes, H. Möllmann und J. Reischi

Aus dem Institut für Sportmedizin (Direktor: Prof. Dr. E.J. Klaus) und dem Anatomischen Institut der Universität Münster (II. ordentlicher Lebrstubl: Prof. Dr. H. Knocbe)

(Eingegangen am 29. September 1967)

Der Nachweis von Doping-Mitteln eifolgt heute vorwiegend durch Analyse von Urinproben. Jedoch ist eine verläßliche Harnentnahme durch den Probanden nicht immer gewährleistet, wie z. B. Täuschungsversuche durch Abgabe von Fremdharn zeigen.

Auch der Nachweis von Dopingmitteln im Blut stößt wegen der Entnahmetechnik bei den zu untersuchenden Personen auf erhebliche Schwierigkeiten, zumal eine Blutprobe bei den derzeitigen gesetzlichen Bestimmungen nicht immer gefordert werden kann. Außerdem ist dieses Verfahren mit einem größeren technischen Aufwand verbunden. Es sollte daher cine gut überprüfbare und leicht ausführbare Nachweismethode angestrebt werden.

Uberraschenderweise wurde ein Nachweis von stimulierenden Substanzen im Speichel bisher wenig durchgeführt (1). Dabei ergäben sich bei der Untersuchung dieser Gewebsflüssigkeit wesentliche Vorteile, die sowohl in der Möglichkeit einer raschen Entnahme wie auch in einer guten Kontrollierbarkeit der zu untersuchenden Personen liegen. Ziel dieser Untersuchungen war es daher, einen verläßlichen Nachweis von Dopingmitteln aus dem Speichel zu finden.

Die Versuche beschränkten sich zunächst auf eines der gebräuchlichsten Dopingmittel, D-Methamphetamin (Pervitin). Für die chemische Identifizierung stehen einfach durchzuführende Farb-

1) 1-Phenyl-2-methylaminopropan.

2) I. Mitteilung: Sportarzt Köln 18, 361 (1967). reaktionen zur Verfügung. Besonders eignete sich eine Modifikation der Methode von FEIGL (2), die mit körpereigenen Aminen ähnlicher Struktur keine Reaktion zeigt.

Der Nachweis beruht auf der Bildung eines in organischenLösungsmitteln löslichen Kupfersalzes einer Dithiocarbaminsäure, das bei Einwirkung von Kupferionen, Schwefelkohlenstoff und Ammoniak auf methamphetaminhaltige Lösungen entsteht:
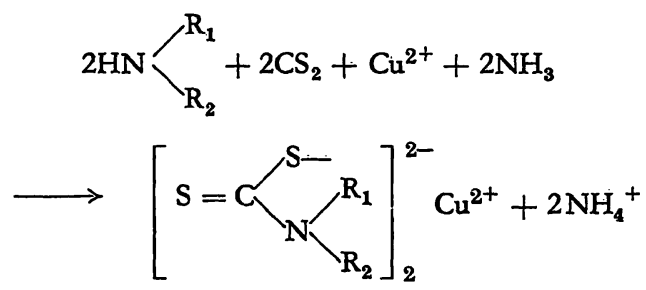

Mit diesem Vetfahten läßt sich Methamphetamin nach Gabe von $15 \mathrm{mg}$ pro $70 \mathrm{~kg}$ Körpergewicht bereits nach 15-30 Min. im Speichel nachweisen. Der positive Ausfall der Reaktion hält noch mindestens $36 \mathrm{Stdn}$. an. Diese Untersuchungsergebnisse stehen in Übereinstimmung zu früheren Befunden (3), erlauben jedoch einen schnelleren Nachweis mit geringerem Aufwand. Daher gestattet diese Methode auch eine rasche und einfache Kontrolle am Wettkampfort.

\section{Methodik}

Es werden $0,2 \mathrm{mg}$ D-Methamphetamin pro $\mathrm{kg}$ Körpergewicht in einer Steckkapsel gegeben, die nach 4-5 Min. Verweildauer im - Magen zerfällt (4). Speichel wurde durch Ausspucken oder durch Umspülen mit Wasser entnommen. Die Probe wurde mit $1,0 \mathrm{~m} /$ 5proz. Kupfersulfatlösüng und 10 Tropfen konz. Ammoniak versetzt. Dann wurde mit einem Gemisch aus Chloroform : Schwefelkohlenstoff $(3: 0,01 \mathrm{v} / \mathrm{v}) 5 \mathrm{Min}$. ausgeschüttelt, die Probe zentrifugiert, die Schichten getrennt, die Chloroformphase filttiert und nach dem Trocknen mit Natriumsulfat visuell oder im Photometer gegen eine Blindprobe verglichen. Es tritt bei positivem Ausfall eine gelbe bis bräunliche Färbung der organischen Phase ein. 